\title{
Tracking Transplanted Stem Cells Using Magnetic Resonance Imaging and the Nanoparticle Labeling Method in Urology
}

\author{
Jae Heon Kim, ${ }^{1}$ Hong J. Lee, ${ }^{2}$ and Yun Seob Song ${ }^{1}$ \\ ${ }^{1}$ Department of Urology, Soonchunhyang University Hospital, College of Medicine, Soonchunhyang University, Seoul, Republic of Korea \\ ${ }^{2}$ Biomedical Research Institute, Chung-Ang School of Medicine, Seoul, Republic of Korea \\ Correspondence should be addressed to Hong J. Lee; leehj71@gmail.com and Yun Seob Song; yssong@schmc.ac.kr
}

Received 4 September 2014; Revised 10 March 2015; Accepted 17 March 2015

Academic Editor: Zhengchao Dong

Copyright (c) 2015 Jae Heon Kim et al. This is an open access article distributed under the Creative Commons Attribution License, which permits unrestricted use, distribution, and reproduction in any medium, provided the original work is properly cited.

\begin{abstract}
A reliable in vivo imaging method to localize transplanted cells and monitor their viability would enable a systematic investigation of cell therapy. Most stem cell transplantation studies have used immunohistological staining, which does not provide information about the migration of transplanted cells in vivo in the same host. Molecular imaging visualizes targeted cells in a living host, which enables determining the biological processes occurring in transplanted stem cells. Molecular imaging with labeled nanoparticles provides the opportunity to monitor transplanted cells noninvasively without sacrifice and to repeatedly evaluate them. Among several molecular imaging techniques, magnetic resonance imaging (MRI) provides high resolution and sensitivity of transplanted cells. MRI is a powerful noninvasive imaging modality with excellent image resolution for studying cellular dynamics. Several types of nanoparticles including superparamagnetic iron oxide nanoparticles and magnetic nanoparticles have been used to magnetically label stem cells and monitor viability by MRI in the urologic field. This review focuses on the current role and limitations of MRI with labeled nanoparticles for tracking transplanted stem cells in urology.
\end{abstract}

\section{Introduction}

Molecular imaging technologies have evolved recently and facilitate functional monitoring and evaluation of genes and organs for their roles in health and disease $[1,2]$. Stem cell transplantation has good prospects for clinical application. However, the challenges in molecular imaging are to develop effective imaging strategies with a combination of imaging modalities, labeling reporter systems, and probes. Several studies have used magnetic resonance imaging (MRI) to trace transplanted stem cells in animal models $[3,4]$.

Several molecular imaging modalities including positron emission tomography (PET), MRI, and newer modalities are based on transmitting light through tissues, such as in vivo bioluminescence imaging and fluorescence imaging. Among them, MRI is the most popular imaging modality. MRI used in conjunction with magnetically labeling is a powerful technique for noninvasively detecting and tracking transplanted cells in longitudinal animal studies $[1,2,5]$.

Labeling materials have great importance in the field of molecular imaging. Labeling stem cells makes merged cells distinguishable from host cells to follow transplanted stem cells.

Molecular materials for labeling should first reveal cellular and molecular processes throughout the entire study period. Secondly, the probes should be highly sensitive to small changes in cell function and distribution. Finally, they should not significantly alter the labeled biological process itself $[1,2,5]$.

Gadolinium and ferric oxide are two common cell labeling contrast media used during MRI [6]. New technologies with tumor targeting and drug delivery are being conceptualized. Developments in nanotechnology have provided more innovative and effective approaches in various areas of clinical research, such as diagnosis [7], monitoring [8, 9], and therapy [10-12]. Labeling with nanoparticles is an emerging trend, particularly in oncology such as "cancer nanotheranostics," which includes simultaneous imaging and treating cancer cells by applying nanoparticles [13].

Although many studies have investigated the efficiency of molecular imaging using MRI with labeled nanoparticles, few studies are available in the urologic field. The aim of 


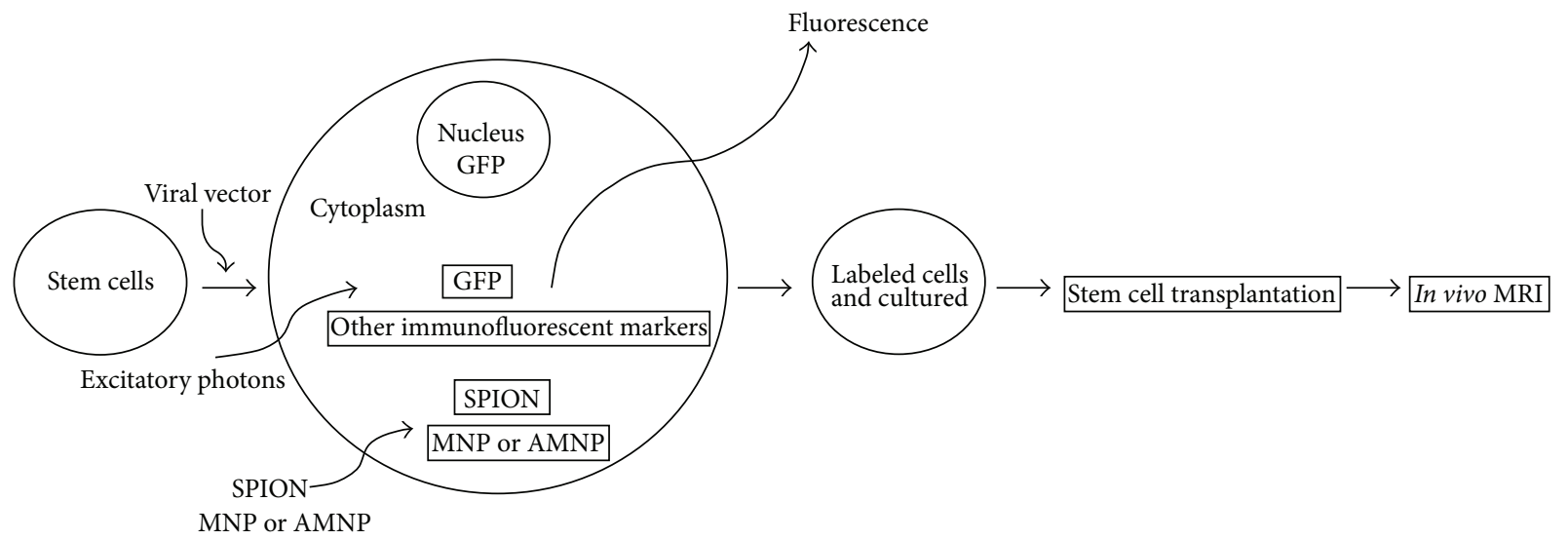

FIGURE 1: Schematic imaging of stem cell tracking using magnetic resonance imaging (MRI) combined with nanoparticle labeling.

this study was to review MRI and labeling techniques for tracking transplanted stem cells (physiological labeling) in the urologic field and to review the characteristics and limitations of current nanoparticle labeling methods.

\section{Stem Cell Labeling}

Cell labeling can be divided into physical cell labeling and reporter gene imaging. Physical cell labeling is completed before cell administration and can be accomplished with superparamagnetic iron oxide (SPIO) particles for MRI [14, 15] and radionuclide labeling for single-photon emission computed tomography [16] and PET [17]. In reporter gene imaging, a gene coding synthesis of a detectable protein is introduced into a target cell line or tissue via viral or nonviral vectors. As a result, changes in signals following cell administration can be used as indicators of cell proliferation and death [18].

Many labeling techniques involve incubating cells and use of transfection agents. The different magnetic labeling techniques result in a considerable increase in the cellular iron content [26], which is 100 times greater than physiological levels [27]. The largest amount of intracellular iron oxide particles and the use of high-resolution gradient echo sequences allow for highly sensitive in vivo MRI methods for detecting viability and efficiency of transplanted stem cells.

Most studies have tracked physically labeled transplanted stem cells (Figure 1). Migration of lymphocytes [28], hematopoietic stem cells [29], mesenchymal stromal cells (MSCs) [30], neuronal precursor cells [31], and tumor cells [32] has been demonstrated in different disease models using in vivo MRI. Specific cancer stem cell antigens or receptors have been detected by reporter gene imaging.

Application of conventional GFP-like fluorescent proteins, including eGFP, DsRed, and mCherry, has limitations due to the penetration depths of visible light in the body [33]. To overcome this limitation, near-infrared fluorescent protein (IFP) has been developed from the DrBphP bacterial phytochrome of Deinococcus radiodurans and showed the possibility in the application of IFPs for protein labeling and in vivo tracking imaging [5].

\section{Iron Oxide Nanoparticles}

The original iron oxide nanoparticles were developed in 1995 and were Dextran-coated iron oxide nanoparticle with a $100-150 \mathrm{~nm}$ hydrodynamic radius and contained a 5-10 nm iron oxide core [34]. These standard, well-characterized iron oxide nanoparticles have been used widely, but low labeling amounts and efficiencies were shortcomings [35]. To overcome this weak point, simple transfection agents were combined with ferumoxides enabling robust labeling of a number of cell types [36].

Most recently, a nanomaterial consisting of a mixture of ferumoxytol, heparin sulfate, and protamine sulfate has been reported and can be used to safely label various types of cells for tracking by MRI [8]. Ferumoxytol, heparin sulfate, and a protamine sulfate conjugate is currently the most popular material for physical cell labeling in urology [37]. Dextrancoated superparamagnetic iron oxide nanoparticles (SPI$\mathrm{ONs}$ ) and micron-sized iron oxide particles were developed for MRI-based cell tracking $[26,38]$. The size of iron oxide particles for cell labeling ranges from very small particles to micron-sized particles, and SPIO is a medium-sized particle [39-41].

Among the different types of nanoparticles, SPIONs are promising candidates for use with molecular imaging modalities due to their superparamagnetic behavior and surfacemodification properties. One of the important features of SPIONs is that they lose their magnetism and become highly dispersed when the magnetic field is switched off, which prevents easy recognition and engulfment by macrophages [42].

As SPIONs are biodegradable and biocompatible, they can be applied in various biomedical fields, such as magnetofection [43], gene therapy [44], and cell and biological material separation [45]. SPIONs are mostly magnetite $\left(\mathrm{Fe}_{3} \mathrm{O}_{4}\right)$, and they convert to maghemite when exposed to oxygen. 
They can be metabolized easily and transported by proteins, such as ferritin, transferrin, and hemosiderin, and they can be stored in endogenous iron reserves of the body for later use $[1,2]$.

The advantage of applying a magnetic field to guide nanoparticles to their target is to reduce stem cell waste, lower the frequency of stem cell administration, and avoid unwanted side effects $[1,2]$. SPIONs are very promising materials for biomedical applications due to their increased ability to covalently attach to various receptors, peptides, antibodies, or ligands [46]. Furthermore, SPIO particles have no adverse effects on viability or proliferation of labeled cells $[40,47]$.

\section{Non-SPION Nanoparticles}

Most magnetic labeling procedures depend on Dextrancoated magnetic nanoparticles. Several magnetic cell labeling methods have been developed but the most commonly used one is coincubating Dextran-coated nanoparticles with a transfection agent [48].

However, these materials follow a low-efficiency fluidphase endocytosis pathway and require long incubation times or the use of transfection agents to achieve substantial iron uptake. Moreover, the complexes formed by the nanoparticles and transfection agents are not easily controlled.

Anionic magnetic nanoparticles (AMNPs) have negative surface charges, are free of a Dextran coating, adsorb readily to cell membranes, and are internalized without the need for transfection agents or long incubation times [20, 49, 50]. AMNPs permit controlled uptake by various cell types [20, 51-53]. AMNPs have advantages of easy and rapid absorption and subsequent internalization by endocytosis $[50,54,55]$. AMNP biocompatibility has been demonstrated in many preclinical studies, including local cell grafts for tissue regeneration $[20,56]$ and immune cell trafficking after systemic injection $[52,57,58]$.

Fluorescent magnetic nanoparticles (MNPs) contain rhodamine B isothiocyanate within a silica shell to overcome the negative surface charge. This tagging material does not require a transfection agent during cell labeling, the MNP core is composed of ferrite, and the inner silica shell portion contains fluorescent materials [59]. It has both magnetic and optical features, and Prussian blue staining is not necessary to detect viability and efficiency of transplanted stem cells within tissue. In vivo tracking of transplanted MSCs labeled with fluorescent MNPs in a liver cirrhosis rat model by MRI has been reported [60].

Among the six studies that used MRI and physical labeling, three used SPIONs, two used MNPs, and one used AMNPs (Table 1).

Gadolinium (Gd) based contrast agents are normally used to reduce $T 1$ period and to give positive contrast in MR images. Available agents are different kinds of gadolinium ion based chelates which are relatively stable molecules. To date, Gd based contrast agent has evolved to be more efficient contrast agents including $\mathrm{Gd} 3+$ based agents with higher molecular weight like Gd-DTPA functionalized polymers,
Gd-DTPA terminated dendrimers, and Gd complex loaded liposomes as well as high density lipoprotein nanoparticles or micelles [61]. Recently, several new nanoparticles are introduced for in vivo imaging including $\mathrm{Fe}_{3} \mathrm{O}_{4} @ \mathrm{SiO}_{2}$ nanoparticles and Gd-DOTA-peptides [62-64].

\section{Ideal Features of Labeled Nanoparticles}

Nanoparticles for any biological application must be biocompatible, nontoxic, and stable at physiological $\mathrm{pH}$. The ideal features are high magnetization and a narrow size distribution. The nanoparticles should have contrast enhancement properties for imaging and tracking of malignant cells/tissues, and their surfaces should be coated with biodegradable material. They should have the ability to conjugate with a range of receptors with high targeting and drug-delivery efficiencies. The half-life should be long, and the zeta-potential should be optimized $[1,5,9]$.

\section{Current Uses for MRI Techniques in Urology}

MRI is a widely used powerful imaging technique that provides high resolution in the field of urology. It is used to evaluate stem cells transplanted to urologic organs. MRI alone or MRI with a physical labeling method has been used to monitor the efficiency of cell transplantation, cellular homing, and targeting. MRI has been used in prostate cancer research, bladder dysfunction research, urethral sphincter studies, and a penis study (Table 1).

6.1. Prostate Cancer. Molecular imaging combined with a labeling technique has been used to detect specific prostate cancer antigens. Two studies showed the efficiency of MRI for detecting transplanted stem cells as a vector for prodrug therapy. Abrate et al. [21] reported that MRI can be used to follow orthotopic tumor progression. Although those authors did not apply a physical labeling method, they demonstrated that intravenous injections of CD-MSC cells, followed by intraperitoneal administration of 5-fluorocytosine, caused tumor regression in transgenic adenocarcinoma of the mouse prostate model, which develops aggressive and spontaneous prostate cancer.

Lee et al. [19] reported monitoring the migration of genetically modified stem cells by MRI after labeling the cells with fluorescent MNPs. Human neural stem cells encoding CD (HB1.F3.CD) were prepared and labeled with MNPs (Figure 2). HB1.F3.CD stem cells systemically transplanted into tumor-bearing C57B mice migrated toward the tumor, and tumor implant volume decreased significantly in combination with the prodrug 5-FC.

6.2. Bladder. Traditionally, many studies showed the efficacy of stem cell treatment in bladder dysfunction [65-67]; however only several studies introduced molecular imaging techniques. Yun and Ja [24] showed similar viability of MSCs loaded with SPIONs compared to unlabeled cells. SPIOlabeled MSCs underwent normal chondrogenic, adipogenic, 


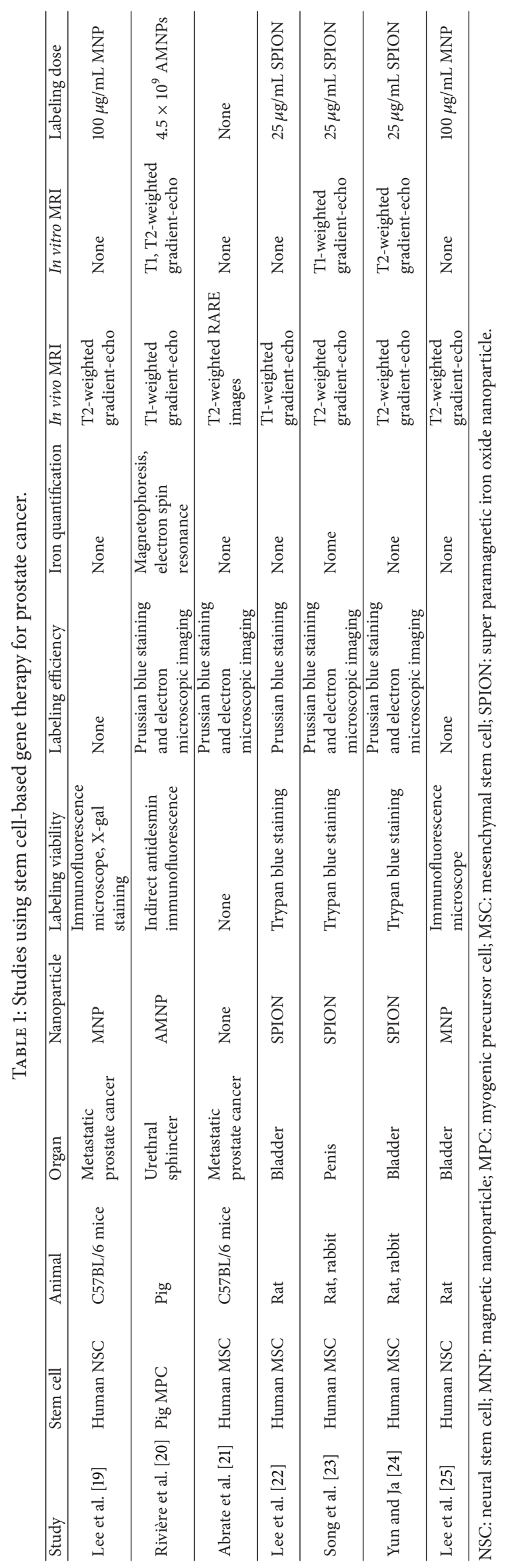



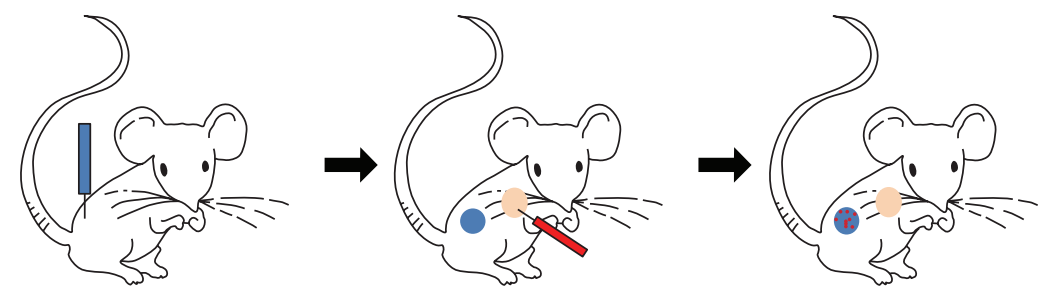

(a)

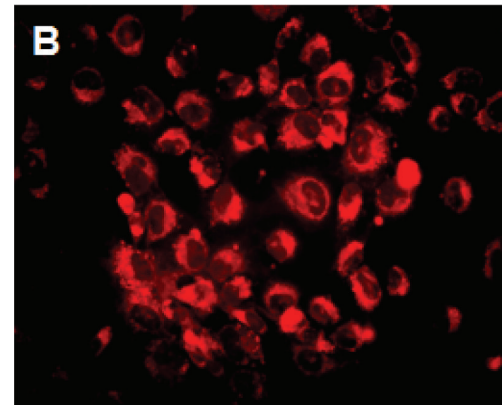

(b)

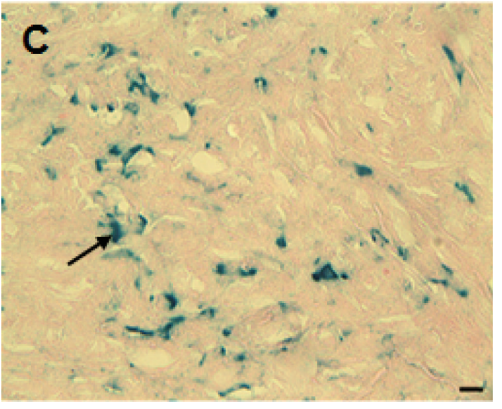

(c)

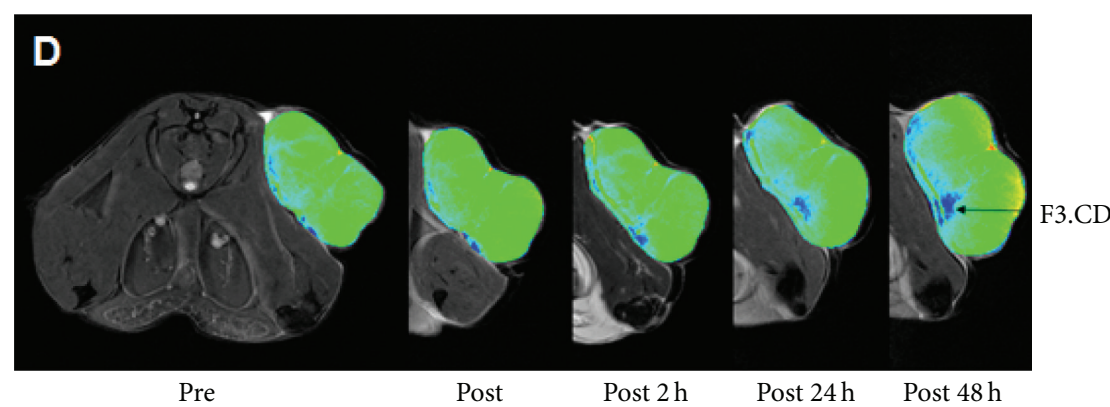

(d)

FIGURE 2: Intravascular delivery of stem cells (HB1.F3.CD) targeting prostate cancer. (a) Schematic illustration of inducing prostate cancer using TRAMPC2, a systemic injection of HB1.F3.CD cells, and migration of the gene-modified stem cells toward the prostate cancer. Blue $=$ TRAMPC2; red = HB1.F3.CD cells. (b) X-gal staining of induced prostate cancer 2 days after injecting HB1.F3.CD cells. Arrow indicates the cells. (c) Magnetic resonance imaging (MRI) of prostate cancer $48 \mathrm{hr}$ after injecting HB1.F3.CD cells into mice. Arrow indicates the cells (scale bar, $100 \mu \mathrm{m})$. HB1.F3, neural stem cells; CD, cytosine deaminase.

and osteogenic differentiation. MRI signal intensity in the areas of SPION-labeled MSCs in rat and rabbit bladders decreased and was confined locally. MRI demonstrated that SPION-labeled MSCs injected into the bladder could be seen for at least 12 weeks.

Lee et al. [22] reported that MRI images were useful to track transplanted MSCs in bladder outlet obstruction induced bladder dysfunction. Serial T2-weighted MRI images were taken immediately after transplant of SPIONlabeled MSCs and at 4 weeks after transplantation. T2weighted MRI showed a clear hypointense signal induced by the SPION-labeled MSCs. Collagen and transforming growth factor- $\beta$ expression protein increased after bladder outlet obstruction, and the expression of both returned to original levels after MSC transplantation.

Lee et al. [25] reported the efficiency of MRI for tracking transplanted MSCs in a spinal cord injury-induced bladder dysfunction model. MNP-labeled B10 cells were injected into the bladder wall 4 weeks after the spinal cord injury. Serial
MRI was taken immediately after MNP-B10 injection and at 4 weeks after transplantation.

6.3. Penis. Song et al. [23] suggested that MRI can be used to investigate the long-term therapeutic potential of MSCs to treat erectile dysfunction. SPION-labeled MSCs injected into the corpus cavernosa of rats and rabbits were evaluated noninvasively by molecular MRI. MRI signal intensity at the areas of SPION-labeled MSCs in the rat and rabbit corpus cavernosa decreased and was confined locally. MRI demonstrated that the MSCs could be observed for at least 12 weeks after injection into the corpus cavernosum.

6.4. Urethral Sphincter. Rivière et al. [20] magnetically labeled muscle implants with AMNPs. They evaluated the biocompatibility of the labeling procedure and its utility for noninvasive MRI follow-up of cell therapy in a female pig model. AMNPs were adsorbed onto the implant surface of myogenic precursor cells and were magnetically labeled 
within the implants. Magnetic labeling did not affect cell proliferation or differentiation. Autograft detection in vivo by 0.3-T MRI was possible for up to 1 month.

\section{Discussion}

Although molecular imaging techniques have evolved significantly during the last decade, no single imaging modality can provide all the information required to track transplanted stem cells and monitor their functional effects. Each imaging modality used for stem cell tracing has its advantages and disadvantages $[54,68]$. PET has high sensitivity for tracking biomarkers in vivo but lacks the ability to provide detailed anatomic structure. Optical imaging has high molecular sensitivity but provides less anatomical localization and is mainly used in small animals. MRI coupled with physical labeling has high resolution and the capabilities of threedimensional anatomical imaging. However, MRI has low sensitivity for cell tracing. Moreover, it cannot detect cell number or location by cell division because cell division may dilute intracellular markers with the shedding of iron particles [69]. Hence, it is necessary to combine complementary imaging methodologies as with multimodality imaging approaches.

Some nonspecific signal problems occur with different imaging modalities as a result of dead transplanted cells. For example, dead transplanted cells containing iron oxide nanoparticles may result in MRI signals representing macrophage phagocytosis of labeled cell debris $[1,2,5]$. Limitations continue in the basic knowledge about the pivotal biological characters of transplanted stem cells, such as survival, integration, and migration, and the influence of the host microenvironment. Despite the potential for biomedical applications, SPIONs face some targeting and imaging limitations. The proportion of SPIONs that reach the target through surface-bound antibodies is low, thereby limiting their application for imaging and drug delivery.

MRI is a commonly used imaging modality and it could be used in a large animal model. To detect successful delivery and subsequent migration using iron oxide-based agents, ex vivo labeling of the stem cells are required [1]. The robust negative contrast image generated by iron oxide agents has shown efficient cell labeling. Studies have also reported successful tracking at near single-cell resolution [70]. However, to assess the viability additional techniques are needed such as using promoter genes, engineering cells to overexpress transferrin receptors, and nongenomic technique using manganese-enhanced MRI.

To date, the important pitfalls of MRI with SPIO labeled cells or luciferase-based bioluminescent imaging are that these modalities could provide the information about cell survival, anatomical coregistration of engrafted cells together with real time, and image-guided delivery. To overcome these limitations, chemical exchange saturation transfer is an emerging MRI contrast mechanism based on the use of radiofrequency saturation pulses to detect agents containing protons that exchange rapidly with water [27]. Chan et al. [9] reported that $\mathrm{pH}$ nanosensor-based MRI technique can monitor cell death in vivo noninvasively. They demonstrated that specific MRI parameters related with cell death of microencapsulated hepatocytes are associated with the measured bioluminescence imaging radiance.

To overcome this problem, classic physical labeling must be upgraded with a reporter gene imaging technique. In fact, this combined modality is being investigated in cancer stem cell studies. SPIONs with specific tumor-targeting ligands and sensitive imaging probes must be developed [71]. To date, most in vivo imaging studies showed the limitations in detecting the engraftment of stem cells [72].

Another major problem facing drug delivery using nanoparticles is the burst effect. When drug-coated nanoparticles are injected into a system, a significant quantity of the drug is liberated suddenly due to alterations in the physiological host environment, which can be dangerous to the patient $[1,2,5]$. To overcome this effect, nanoparticles must be cross-linked with polymers or incorporated into a polymer matrix that provides more rigidity and helps provide sustained drug delivery under in vivo conditions for longer times [1, 2, 5]. Mahmoudi and Laurent [73] demonstrated increased stability of drug-loaded SPIONs under in vivo conditions using a PEG-cofumarate cross linking agent. Other issues that must be solved regarding nanoparticles include toxicity, gene alternations, penetration of the bloodbrain barrier, and colloidal stability $[1,2,5]$.

Molecular imaging combined with a nanoparticle labeling method is useful not only for physical labeling to monitor stem cells but also to detect prostate cancer antigens. Recent preclinical studies on multimodal molecular imaging methods have the potential to be helpful for noninvasive prostate cancer diagnosis and image-guided immunotherapy [74, 75]. Multiple groups are actively pursuing the development of imaging probes for cellular and molecular MRI [75-79]. More studies are needed to develop various molecular markers, including ligands, antibodies, and peptides that can easily bind MRO probes.

In vivo imaging represents a dedicated platform to evaluate and quantify molecular and cellular events related to cellular engraftment. This integrative approach should be more developed with validation for systematic translation of stem cell therapy.

\section{Conclusions}

Molecular imaging is a new discipline that allows for in vivo cellular and molecular imaging of pathophysiological processes and the results of therapeutic interventions. MRI is a contending and complementing modality for stem cell studies in urology. MRI can be used to evaluate migration and survival of transplanted stem cells in prostate cancer and bladder dysfunction models. It has also shown potential utility for use on erectile dysfunction and urethral sphincter dysfunction. Noninvasive imaging methods using MRI have the advantage of longitudinal monitoring of transplanted stem cells in animals.

\section{Conflict of Interests}

The authors declare that they have no conflict of interests. 


\section{Acknowledgment}

This research was supported by the Research Program through the National Research Foundation of Korea (NRF) funded by the Ministry of Education, Science, and Technology (2010-0011678) and by the Soonchunhyang University Research Fund.

\section{References}

[1] L. Josephson, C.-H. Tung, A. Moore, and R. Weissleder, "Highefficiency intracellular magnetic labeling with novel superparamagnetic-Tat peptide conjugates," Bioconjugate Chemistry, vol. 10, no. 2, pp. 186-191, 1999.

[2] L. Tong, M. Zhao, S. Zhu, and J. Chen, "Synthesis and application of superparamagnetic iron oxide nanoparticles in targeted therapy and imaging of cancer," Frontiers of Medicine in China, vol. 5, no. 4, pp. 379-387, 2011.

[3] L. E. Gonzalez-Lara, X. Xu, K. Hofstetrova, A. Pniak, A. Brown, and P. J. Foster, "In vivo magnetic resonance imaging of spinal cord injury in the mouse.," Journal of Neurotrauma, vol. 26, no. 5, pp. 753-762, 2009.

[4] J. Guenoun, A. Ruggiero, G. Doeswijk et al., "In vivo quantitative assessment of cell viability of gadolinium or iron-labeled cells using MRI and bioluminescence imaging," Contrast Media \& Molecular Imaging, vol. 8, no. 2, pp. 165-174, 2013.

[5] D. Yu, W. C. Gustafson, C. Han et al., "An improved monomeric infrared fluorescent protein for neuronal and tumour brain imaging," Nature Communications, vol. 5, article 4626, 2014.

[6] L. Yan, Y. Han, Y. He et al., "Cell tracing techniques in stem cell transplantation," Stem Cell Reviews, vol. 3, no. 4, pp. 265-269, 2007.

[7] M. V. Yigit, A. Moore, and Z. Medarova, "Magnetic nanoparticles for cancer diagnosis and therapy," Pharmaceutical Research, vol. 29, no. 5, pp. 1180-1188, 2012.

[8] J. E. Rosen, L. Chan, D.-B. Shieh, and F. X. Gu, "Iron oxide nanoparticles for targeted cancer imaging and diagnostics," Nanomedicine: Nanotechnology, Biology, and Medicine, vol. 8, no. 3, pp. 275-290, 2012.

[9] K. W. Y. Chan, G. Liu, X. Song et al., "MRI-detectable pH nanosensors incorporated into hydrogels for in vivo sensing of transplanted-cell viability," Nature Materials, vol. 12, no. 3, pp. 268-275, 2013.

[10] S. Balivada, R. S. Rachakatla, H. Wang et al., "A/C magnetic hyperthermia of melanoma mediated by iron(0)/iron oxide core/shell magnetic nanoparticles: a mouse study," BMC Cancer, vol. 10, article 119, 2010.

[11] F. Dilnawaz, A. Singh, C. Mohanty, and S. K. Sahoo, "Dual drug loaded superparamagnetic iron oxide nanoparticles for targeted cancer therapy," Biomaterials, vol. 31, no. 13, pp. 3694-3706, 2010.

[12] A. Z. Wang, V. Bagalkot, C. C. Vasilliou et al., "Superparamagnetic iron oxide nanoparticle-aptamer bioconjugates for combined prostate cancer imaging and therapy," ChemMedChem, vol. 3, no. 9, pp. 1311-1315, 2008.

[13] P. Zou, Y. Yu, Y. A. Wang et al., "Superparamagnetic iron oxide nanotheranostics for targeted cancer cell imaging and $\mathrm{pH}-$ dependent intracellular drug release," Molecular Pharmaceutics, vol. 7, no. 6, pp. 1974-1984, 2010.

[14] E. Syková and P. Jendelová, "Magnetic resonance tracking of transplanted stem cells in rat brain and spinal cord," Neurodegenerative Diseases, vol. 3, no. 1-2, pp. 62-67, 2006.
[15] V. Vanecek, V. Zablotskii, S. Forostyak et al., "Highly efficient magnetic targeting of mesenchymal stem cells in spinal cord injury," International Journal of Nanomedicine, vol. 7, pp. 37193730, 2012.

[16] A. S. Arbab, C. Thiffault, B. Navia et al., "Tracking of In111-labeled human umbilical tissue-derived cells (hUTC) in a rat model of cerebral ischemia using SPECT imaging," $B M C$ Medical Imaging, vol. 12, article 33, 2012.

[17] J. K. Jaiswal, H. Mattoussi, J. M. Mauro, and S. M. Simon, "Longterm multiple color imaging of live cells using quantum dot bioconjugates," Nature Biotechnology, vol. 21, no. 1, pp. 47-51, 2003.

[18] N. Sun, A. Lee, and J. C. Wu, "Long term non-invasive imaging of embryonic stem cells using reporter genes," Nature Protocols, vol. 4, no. 8, pp. 1192-1201, 2009.

[19] H. J. Lee, S. W. Doo, D. H. Kim et al., "Cytosine deaminaseexpressing human neural stem cells inhibit tumor growth in prostate cancer-bearing mice," Cancer Letters, vol. 335, no. 1, pp. 58-65, 2013.

[20] C. Rivière, F. P. Boudghène, F. Gazeau et al., "Iron oxide nanoparticle-labeled rat smooth muscle cells: cardiac MR imaging for cell graft monitoring and quantitation," Radiology, vol. 235, no. 3, pp. 959-967, 2005.

[21] A. Abrate, R. Buono, T. Canu et al., "Mesenchymal stem cells expressing therapeutic genes induce autochthonous prostate tumour regression," European Journal of Cancer, vol. 50, no. 14, pp. 2478-2488, 2014.

[22] H. J. Lee, J. H. Won, S. H. Doo et al., "Inhibition of collagen deposit in obstructed rat bladder outlet by transplantation of superparamagnetic iron oxide-labeled human mesenchymal stem cells as monitored by molecular magnetic resonance imaging (MRI)," Cell Transplantation, vol. 21, no. 5, pp. 959-970, 2012.

[23] Y.-S. Song, J.-H. Ku, E.-S. Song et al., "Magnetic resonance evaluation of human mesenchymal stem cells in corpus cavernosa of rats and rabbits," Asian Journal of Andrology, vol. 9, no. 3, pp. 361-367, 2007.

[24] S. S. Yun and H. K. Ja, "Monitoring transplanted human mesenchymal stem cells in rat and rabbit bladders using molecular magnetic resonance imaging," Neurourology and Urodynamics, vol. 26, no. 4, pp. 584-593, 2007.

[25] H. J. Lee, J. An, S. W. Doo et al., "Improvement in spinal cord injury-induced bladder fibrosis using mesenchymal stem cell transplantation into the bladder wall," Cell Transplantation, 2014.

[26] E. M. Shapiro, S. Skrtic, and A. P. Koretsky, "Sizing it up: cellular MRI using micron-sized iron oxide particles," Magnetic Resonance in Medicine, vol. 53, no. 2, pp. 329-338, 2005.

[27] R. Sun, J. Dittrich, M. Le-Huu et al., "Physical and biological characterization of superparamagnetic iron oxide- and ultrasmall superparamagnetic iron oxide-labeled cells: a comparison," Investigative Radiology, vol. 40, no. 8, pp. 504-513, 2005.

[28] S. A. Anderson, J. Shukaliak-Quandt, E. K. Jordan et al., "Magnetic resonance imaging of labeled T-cells in a mouse model of multiple sclerosis," Annals of Neurology, vol. 55, no. 5, pp. 654-659, 2004.

[29] A. S. Arbab, V. Frenkel, S. D. Pandit et al., "Magnetic resonance imaging and confocal microscopy studies of magnetically labeled endothelial progenitor cells trafficking to sites of tumor angiogenesis," Stem Cells, vol. 24, no. 3, pp. 671-678, 2006.

[30] C. Bos, Y. Delmas, A. Desmoulière et al., "In vivo MR imaging of intravascularly injected magnetically labeled mesenchymal 
stem cells in rat kidney and liver," Radiology, vol. 233, no. 3, pp. 781-789, 2004.

[31] E. M. Shapiro, O. Gonzalez-Perez, J. Manuel García-Verdugo, A. Alvarez-Buylla, and A. P. Koretsky, "Magnetic resonance imaging of the migration of neuronal precursors generated in the adult rodent brain," NeuroImage, vol. 32, no. 3, pp. 1150-1157, 2006.

[32] C. Heyn, J. A. Ronald, S. S. Ramadan et al., "In vivo MRI of cancer cell fate at the single-cell level in a mouse model of breast cancer metastasis to the brain," Magnetic Resonance in Medicine, vol. 56, no. 5, pp. 1001-1010, 2006.

[33] J. Lecoq and M. J. Schnitzer, "An infrared fluorescent protein for deeper imaging," Nature Biotechnology, vol. 29, no. 8, pp. 715716, 2011.

[34] J. W. M. Bulte and D. L. Kraitchman, "Iron oxide MR contrast agents for molecular and cellular imaging," $N M R$ in Biomedicine, vol. 17, no. 7, pp. 484-499, 2004.

[35] A. S. Arbab, L. A. Bashaw, B. R. Miller, E. K. Jordan, J. W. M. Bulte, and J. A. Frank, "Intracytoplasmic tagging of cells with ferumoxides and transfection agent for cellular magnetic resonance imaging after cell transplantation: methods and techniques," Transplantation, vol. 76, no. 7, pp. 1123-1130, 2003.

[36] J. A. Frank, H. Zywicke, E. K. Jordan et al., "Magnetic intracellular labeling of mammalian cells by combining (FDAapproved) superparamagnetic iron oxide MR contrast agents and commonly used transfection agents," Academic Radiology, vol. 9, supplement 2, pp. S484-S487, 2002.

[37] M. Gutova, J. A. Frank, M. D’Apuzzo et al., "Magnetic resonance imaging tracking of ferumoxytol-labeled human neural stem cells: studies leading to clinical use," Stem Cells Translational Medicine, vol. 2, no. 10, pp. 766-775, 2013.

[38] K. A. Hinds, J. M. Hill, E. M. Shapiro et al., "Highly efficient endosomal labeling of progenitor and stem cells with large magnetic particles allows magnetic resonance imaging of single cells," Blood, vol. 102, no. 3, pp. 867-872, 2003.

[39] J. W. M. Bulte, I. D. Duncan, and J. A. Frank, "In vivo magnetic resonance tracking of magnetically labeled cells after transplantation," Journal of Cerebral Blood Flow \& Metabolism, vol. 22, no. 8, pp. 899-907, 2002.

[40] L. Kostura, D. L. Kraitchman, A. M. Mackay, M. F. Pittenger, and J. M. W. Bulte, "Feridex labeling of mesenchymal stem cells inhibits chondrogenesis but not adipogenesis or osteogenesis," NMR in Biomedicine, vol. 17, no. 7, pp. 513-517, 2004.

[41] L. Matuszewski, T. Persigehl, A. Wall et al., "Cell tagging with clinically approved iron oxides: feasibility and effect of lipofection, particle size, and surface coating on labeling efficiency," Radiology, vol. 235, no. 1, pp. 155-161, 2005.

[42] L.-S. Wang, M.-C. Chuang, and J.-A. A. Ho, "Nanotheranostics-a review of recent publications," International Journal of Nanomedicine, vol. 7, pp. 4679-4695, 2012.

[43] S. Prijic, L. Prosen, M. Cemazar et al., "Surface modified magnetic nanoparticles for immuno-gene therapy of murine mammary adenocarcinoma," Biomaterials, vol. 33, no. 17, pp. 4379-4391, 2012.

[44] H. J. Lee, Y. T. C. Nguyen, M. Muthiah et al., "MR traceable delivery of p53 tumor suppressor gene by PEI-functionalized superparamagnetic iron oxide nanoparticles," Journal of Biomedical Nanotechnology, vol. 8, no. 3, pp. 361-371, 2012.

[45] H. Xu, Z. P. Aguilar, L. Yang et al., "Antibody conjugated magnetic iron oxide nanoparticles for cancer cell separation in fresh whole blood," Biomaterials, vol. 32, no. 36, pp. 9758-9765, 2011.
[46] C. Fang, F. M. Kievit, O. Veiseh et al., "Fabrication of magnetic nanoparticles with controllable drug loading and release through a simple assembly approach," Journal of Controlled Release, vol. 162, no. 1, pp. 233-241, 2012.

[47] J. W. M. Bulte, T. Douglas, B. Witwer et al., "Magnetodendrimers allow endosomal magnetic labeling and in vivo tracking of stem cells," Nature Biotechnology, vol. 19, no. 12, pp. 1141-1147, 2001.

[48] J. A. Frank, B. R. Miller, A. S. Arbab et al., "Clinically applicable labeling of mammalian and stem cells by combining superparamagnetic iron oxides and transfection agents," Radiology, vol. 228, no. 2, pp. 480-487, 2003.

[49] C. Wilhelm and F. Gazeau, "Universal cell labelling with anionic magnetic nanoparticles," Biomaterials, vol. 29, no. 22, pp. 3161$3174,2008$.

[50] C. Wilhelm, F. Gazeau, J. Roger, J. N. Pons, and J.-C. Bacri, "Interaction of anionic super-paramagnetic nanoparticles with cells: kinetic analyses of membrane adsorption and subsequent internalization," Langmuir, vol. 18, no. 21, pp. 8148-8155, 2002.

[51] A. Naveau, P. Smirnov, C. Ménager et al., "Phenotypic study of human gingival fibroblasts labeled with superparamagnetic anionic nanoparticles," Journal of Periodontology, vol. 77, no. 2, pp. 238-247, 2006.

[52] P. Smirnov, F. Gazeau, M. Lewin et al., "In vivo cellular imaging of magnetically labeled hybridomas in the spleen with a 1.5-T clinical MRI system," Magnetic Resonance in Medicine, vol. 52, no. 1, pp. 73-79, 2004.

[53] C. Wilhelm, L. Bal, P. Smirnov et al., "Magnetic control of vascular network formation with magnetically labeled endothelial progenitor cells," Biomaterials, vol. 28, no. 26, pp. 3797-3806, 2007.

[54] M. Modo, M. Hoehn, and J. W. M. Bulte, "Cellular MR imaging," Molecular Imaging, vol. 4, no. 3, pp. 143-164, 2005.

[55] C. Wilhelm, C. Billotey, J. Roger, J. N. Pons, J.-C. Bacri, and F. Gazeau, "Intracellular uptake of anionic superparamagnetic nanoparticles as a function of their surface coating," Biomaterials, vol. 24, no. 6, pp. 1001-1011, 2003.

[56] J.-F. Deux, J. Dai, C. Rivière et al., "Aortic aneurysms in a rat model: in vivo MR imaging of endovascular cell therapy," Radiology, vol. 246, no. 1, pp. 185-192, 2008.

[57] C. Billotey, C. Aspord, O. Beuf et al., "T-cell homing to the pancreas in autoimmune mouse models of diabetes: in vivo MR imaging," Radiology, vol. 236, no. 2, pp. 579-587, 2005.

[58] P. Smirnov, E. Lavergne, F. Gazeau et al., "In vivo cellular imaging of lymphocyte trafficking by MRI: a tumor model approach to cell-based anticancer therapy," Magnetic Resonance in Medicine, vol. 56, no. 3, pp. 498-508, 2006.

[59] J. S. Kim, T. J. Yoon, K. N. Yu et al., "Toxicity and tissue distribution of magnetic nanoparticles in mice," Toxicological Sciences, vol. 89, no. 1, pp. 338-347, 2006.

[60] T. H. Kim, J. K. Kim, W. Shim, S. Y. Kim, T. J. Park, and J. Y. Jung, "Tracking of transplanted mesenchymal stem cells labeled with fluorescent magnetic nanoparticle in liver cirrhosis rat model with 3-T MRI," Magnetic Resonance Imaging, vol. 28, no. 7, pp. 1004-1013, 2010.

[61] J.-L. Bridot, A.-C. Faure, S. Laurent et al., "Hybrid gadolinium oxide nanoparticles: multimodal contrast agents for in vivo imaging," Journal of the American Chemical Society, vol. 129, no. 16, pp. 5076-5084, 2007. 
[62] L. Cao, B. Li, P. Yi et al., "The interplay of $T_{1}$ - and $T_{2}$ relaxation on $T_{1}$-weighted MRI of hMSCs induced by GdDOTA-peptides," Biomaterials, vol. 35, no. 13, pp. 4168-4174, 2014.

[63] F. Tian, G. Chen, P. Yi et al., "Fates of $\mathrm{Fe}_{3} \mathrm{O}_{4}$ and $\mathrm{Fe}_{3} \mathrm{O}_{4} @ \mathrm{SiO}_{2}$ nanoparticles in human mesenchymal stem cells assessed by synchrotron radiation-based techniques," Biomaterials, vol. 35, no. 24, pp. 6412-6421, 2014.

[64] P. Yi, G. Chen, H. Zhang et al., "Magnetic resonance imaging of $\mathrm{Fe}_{3} \mathrm{O}_{4} @ \mathrm{SiO}_{2}$-labeled human mesenchymal stem cells in mice at 11.7 T," Biomaterials, vol. 34, no. 12, pp. 3010-3019, 2013.

[65] J. H. Kim, S. R. Shim, S. W. Doo et al., "Bladder recovery by stem cell based cell therapy in the bladder dysfunction induced by spinal cord injury: systematic review and meta-analysis," PLoS ONE, vol. 10, no. 3, Article ID e0113491, 2015.

[66] J. H. Kim, H. J. Lee, and Y. S. Song, "Treatment of bladder dysfunction using stem cell or tissue engineering technique," Korean Journal of Urology, vol. 55, no. 4, pp. 228-238, 2014.

[67] J. H. Kim, S. R. Lee, Y. S. Song, and H. J. Lee, "Stem cell therapy in bladder dysfunction: where are we? and where do we have to go?" BioMed Research International, vol. 2013, Article ID 930713, 10 pages, 2013.

[68] J. C. Sipe, M. Filippi, G. Martino et al., "Method for intracellular magnetic labeling of human mononuclear cells using approved iron contrast agents," Magnetic Resonance Imaging, vol. 17, no. 10, pp. 1521-1523, 1999.

[69] Y. Takahashi, O. Tsuji, G. Kumagai et al., "Comparative study of methods for administering neural stem/progenitor cells to treat spinal cord injury in mice," Cell Transplantation, vol. 20, no. 5, pp. 727-739, 2011.

[70] P. Foster-Gareau, C. Heyn, A. Alejski, and B. K. Rutt, "Imaging single mammalian cells with a $1.5 \mathrm{~T}$ clinical MRI scanner," Magnetic Resonance in Medicine, vol. 49, no. 5, pp. 968-971, 2003.

[71] T. Koyama, M. Shimura, Y. Minemoto et al., "Evaluation of selective tumor detection by clinical magnetic resonance imaging using antibody-conjugated superparamagnetic iron oxide," Journal of Controlled Release, vol. 159, no. 3, pp. 413-418, 2012.

[72] C. Toma, W. R. Wagner, S. Bowry, A. Schwartz, and F. Villanueva, "Fate of culture-expanded mesenchymal stem cells in the microvasculature: in vivo observations of cell kinetics," Circulation Research, vol. 104, no. 3, pp. 398-402, 2009.

[73] M. Mahmoudi and S. Laurent, "Controlling the burst effect of a drug by introducing starch in the structure of magnetic polyurethane microspheres containing superparamagnetic iron oxide nanoparticles," IEEE Transactions on Nanotechnology, vol. 17, pp. 43-51, 2010.

[74] M. Beissert, R. Lorenz, and E. W. Gerharz, "Rational imaging in locally advanced prostate cancer," Der Urologe-Ausgabe A, vol. 47, no. 11, pp. 1405-1416, 2008.

[75] B. Turkbey, P. S. Albert, K. Kurdziel, and P. L. Choyke, "Imaging localized prostate cancer: current approaches and new developments," The American Journal of Roentgenology, vol. 192, no. 6, pp. 1471-1480, 2009.

[76] E. Eyal, B. N. Bloch, N. M. Rofsky et al., "Principal component analysis of dynamic contrast enhanced mri in human prostate cancer," Investigative Radiology, vol. 45, no. 4, pp. 174-181, 2010.

[77] H.-C. Huang, P.-Y. Chang, K. Chang et al., "Formulation of novel lipid-coated magnetic nanoparticles as the probe for in vivo imaging," Journal of Biomedical Science, vol. 16, no. 1, article 86, 2009.
[78] S. E. Lapi, H. Wahnishe, D. Pham et al., "Assessment of an ${ }^{18} \mathrm{~F}-$ labeled phosphoramidate peptidomimetic as a new prostatespecific membrane antigen-targeted imaging agent for prostate cancer," Journal of Nuclear Medicine, vol. 50, no. 12, pp. 20422048, 2009.

[79] Z. Li, L. Jian, H. Wang, and Y. Cui, "Flow injection chemiluminescent determination of clenbuterol using GoldMag particles as carrier," Food Additives and Contaminants, vol. 24, no. 1, pp. 21-25, 2007. 


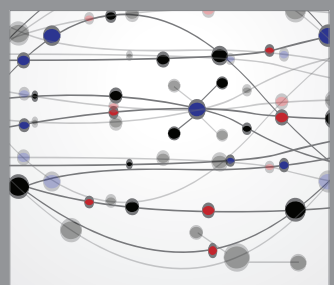

The Scientific World Journal
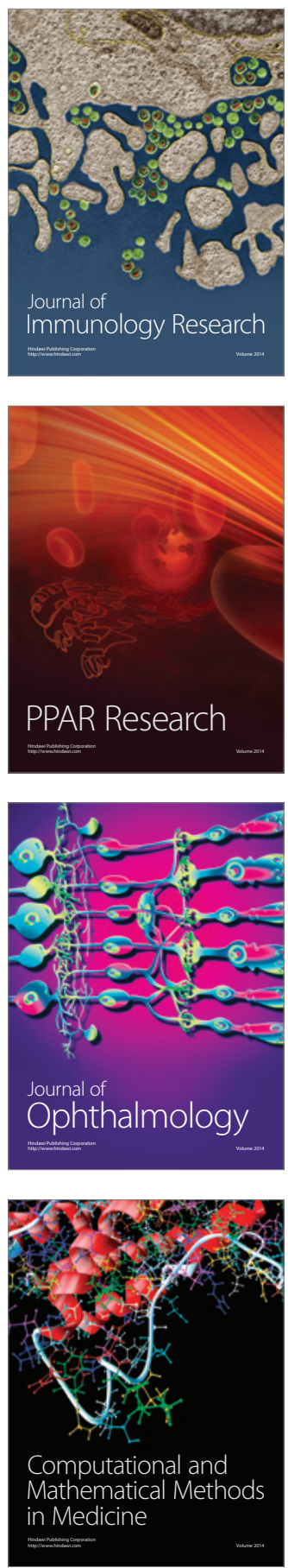

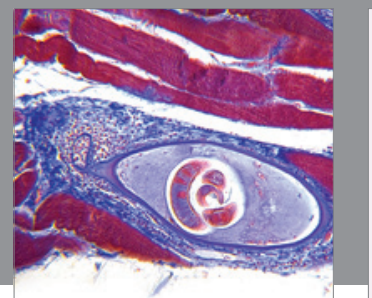

Gastroenterology

Research and Practice
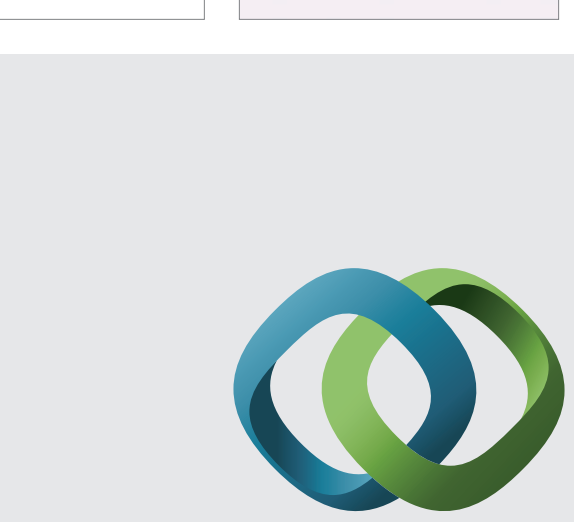

\section{Hindawi}

Submit your manuscripts at

http://www.hindawi.com
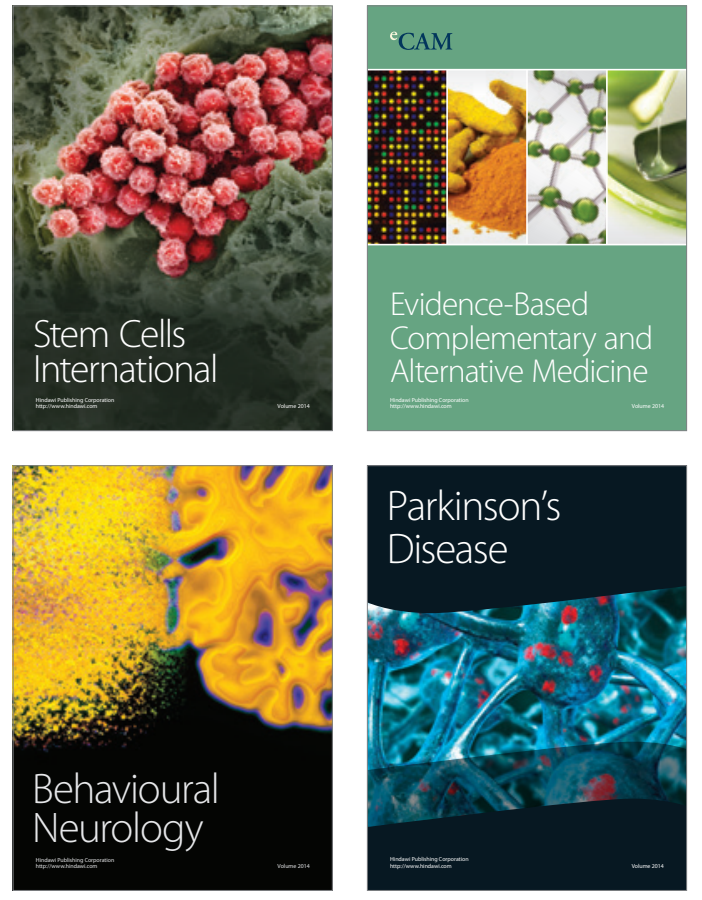
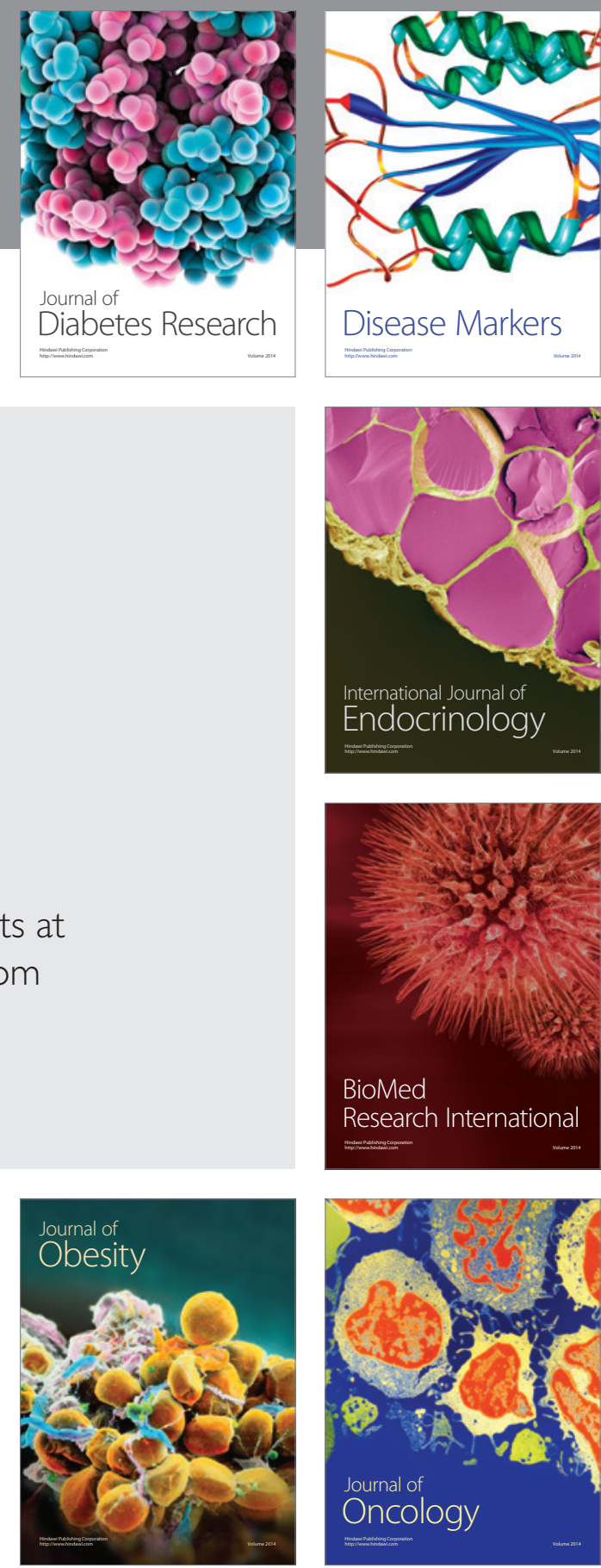

Disease Markers
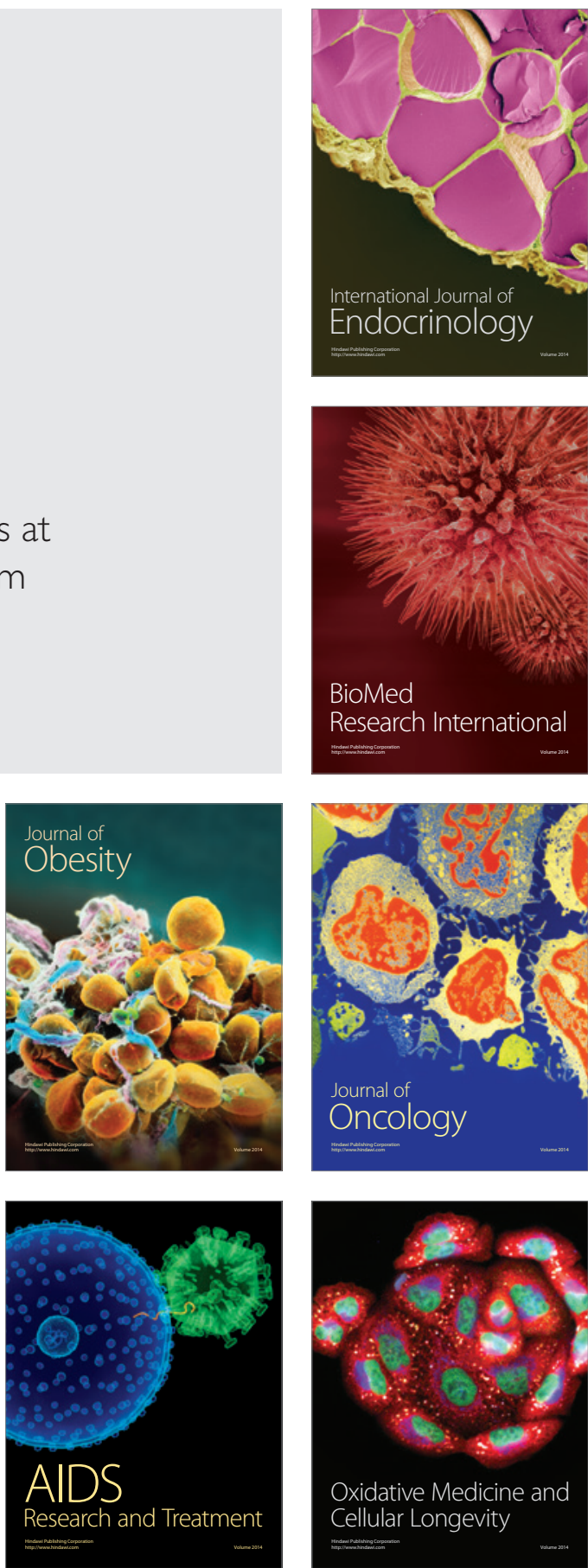